BMJ Open Diabetes Research \& Care

\title{
Glycolytic overload-driven dysfunction of periodontal ligament fibroblasts in high glucose concentration, corrected by glyoxalase 1 inducer
}

\author{
Amal Ashour, ${ }^{1,2}$ Mingzhan Xue, ${ }^{3}$ Maryam Al-Motawa, ${ }^{3,4}$ Paul J Thornalley (1) , 2,3,4 \\ Naila Rabbani (1) ${ }^{5}$
}

To cite: Ashour A, Xue M, Al-Motawa M, et al. Glycolytic overload-driven dysfunction of periodontal ligament fibroblasts in high glucose concentration, corrected by glyoxalase 1 inducer. BMJ Open Diab Res Care 2020;8:e001458. doi:10.1136/ bmjdrc-2020-001458

- Supplemental material is published online only. To view, please visit the journal online (http://dx.doi.org/10.1136/ bmjdrc-2020-001458).

Received 11 April 2020 Revised 23 September 2020 Accepted 26 September 2020

Check for updates

\section{(C) Author(s) (or their} employer(s)) 2020. Re-use permitted under CC BY-NC. No commercial re-use. See rights and permissions. Published by BMJ.

For numbered affiliations see end of article.

Correspondence to Dr Naila Rabbani; n.rabbani@qu.edu.qa

\section{ABSTRACT}

Introduction Patients with diabetes have increased risk of periodontal disease, with increased risk of weakening of periodontal ligament and tooth loss. Periodontal ligament is produced and maintained by periodontal ligament fibroblasts (PDLFs). We hypothesized that metabolic dysfunction of PDLFs in hyperglycemia produces an accumulation of the reactive glycating agent, methylglyoxal (MG), leading to increased formation of the major advanced glycation endproduct, MG-H1 and PDLF dysfunction. The aim of this study was to assess if there is dicarbonyl stress and functional impairment of human PDLFs in primary culture in high glucose concentration-a model of hyperglycemia, to characterize the metabolic drivers of it and explore remedial intervention by the glyoxalase 1 inducer dietary supplement, trans-resveratrol and hesperetin combination (tRES-HESP).

Research design and methods Human PDLFs were incubated in low and high glucose concentration in vitro. Metabolic and enzymatic markers of MG and glucose control were quantified and related changes in the cytoplasmic proteome and cell function-binding to collagen-I, assessed. Reversal of PDLF dysfunction by tRES-HESP was explored.

Results In high glucose concentration cultures, there was a ca. twofold increase in cellular MG, cellular protein MG-H1 content and decreased attachment of PDLFs to collagen-I. This was driven by increased hexokinase-2 linked glucose metabolism and related increased MG formation. Proteomics analysis revealed increased abundance of chaperonins, heat shock proteins (HSPs), Golgi-to-endoplasmic reticulum transport and ubiquitin E3 ligases involved in misfolded protein degradation in high glucose concentration, consistent with activation of the unfolded protein response by increased misfolded MG-modified proteins. PDLF dysfunction was corrected by tRES-HESP.

Conclusions Increased hexokinase-2 linked glucose metabolism produces dicarbonyl stress, increased MG-modified protein, activation of the unfolded protein response and functional impairment of PDLFs in high glucose concentration. tRES-HESP resolves this at source by correcting increased glucose metabolism and may be of benefit in prevention of diabetic periodontal disease.

\section{Significance of this study}

What is already known about this subject?

$\checkmark$ In experimental and clinical diabetes, the periodontal ligament is weakened with disordered collagen fibrils and detachment of periodontal ligament fibroblasts (PDLFs)

- Increased formation of methylglyoxal (MG) occurs in vascular endothelial cells incubated in high glucose concentration associated with hexokinase-2 (HK2)linked glycolytic overload, leading to increased MGmodified proteins, activation of the unfolded protein response and cell detachment from the extracellular matrix; with dysfunction expected in PDLFs.

What are the new findings?

- High glucose concentration imposed dicarbonyl stress on human PDLFs in vitro, increasing the cellular concentration of MG, leading to increased MG modification and related misfolding of cell protein, a proteomic response to cell stress and weakened binding of PDLFs to collagen-I.

- The cytosolic proteome in dicarbonyl stress had increased protein abundance focused on pathways of chaperonins, cellular responses to stress, including response to heat stress - involving heat shock protein and Golgi-to-endoplasmic reticulum retrograde traffic of proteins.

- Dicarbonyl stress was driven by HK2-linked glycolytic overload and proteolytic decrease of glyoxalase 1; and it was prevented by treatment of PDLFs with trans-resveratrol and hesperetin in combination (tRES-HESP).

\section{How might these results change the focus of} research or clinical practice?

- tRES-HESP resolves PDLF dysfunction in high glucose concentration at source by correcting increased glucose metabolism and is a candidate dietary supplement for evaluation for prevention of diabetic periodontal disease.

\section{INTRODUCTION}

Periodontitis is a common complication of diabetes. It has threefold increased 
prevalence, earlier onset, rapid progression and increased severity of symptoms in patients with diabetes compared with subjects without diabetes. Periodontitis is a chronic inflammatory disease of the supporting structures of the teeth and major cause of tooth loss. ${ }^{12}$ Bacterial infection is the main etiological mediator of periodontitis but studies of identical twins found that $50 \%$ of the variability to periodontitis is associated with host factors. ${ }^{3}$ Pathogenesis is considered to be driven by the host inflammatory response to bacterial infection rather than the damaging effects of bacterial pathogens directly. ${ }^{4}$ In the setting of diabetes, dysfunctional glucose metabolism, known to contribute to the pathobiology of vascular complications of diabetes, ${ }^{5}$ may also exacerbate inflammatory mechanisms promoting the development and progression of periodontitis.

Teeth are held in the socket of the alveolar bone by the periodontal ligament (PDL). PDL is a specialized connective tissue between the root of the tooth and the alveolar bone. The major cells of the PDF are periodontal ligament fibroblasts (PDLFs). PDLFs secrete, remodel, degrade and bind to bundles of collagen fibrils, mostly collagen-I, which provide the tensile strength of the PDL. ${ }^{67}$ Binding of PDLFs to collagen-I is mediated mainly by interaction of cell surface integrins, $\alpha_{2} \beta_{1}$ and $\alpha_{11} \beta_{1}$, through GFOGER motif binding sites of collagen-I. ${ }^{8}$ In experimental and clinical diabetes, the periodontal ligament is weakened with disordered collagen fibrils and PDLF detachment from the extracellular matrix. ${ }^{9}{ }^{10}$ The recent finding of hexokinase-2 (HK2)-linked glycolytic overload and activation of unfolded protein response (UPR) and related increased expression of inflammatory mediators, appear particularly timely to explore for mechanistic association with PDLF dysfunction in the pathobiology of periodontitis. ${ }^{11} 12$

Glucose-induced metabolic dysfunction is a causative factor in development of vascular complications of diabetes. One aspect of this is increased concentration of the reactive glucose-derived dicarbonyl metabolite, methylglyoxal (MG) -also called dicarbonyl stress. ${ }^{13}$ MG is formed mainly by low level degradation of glycolytic intermediates, glyceraldehyde-3-phosphate and dihydroxyacetonephosphate. ${ }^{14}$ The formation of MG is increased proportionate with increased glucose metabolism at peripheral sites associated with HK2-linked glycolytic overload. ${ }^{11}$ Abnormal accumulation of MG leads to increased protein glycation by MG, mainly on arginine residues, forming the major advanced glycation endproduct, hydroimidazolone MG-H1 (figure 1A). Arginine modification produces inactivation and misfolding of proteins, ${ }^{15}$ activation of the UPR and downstream inflammatory signaling ${ }^{12}$ and cell detachment from the extracellular matrix. ${ }^{16}$ In collagen of the extracellular matrix, the arginine residues of GFOGER and RGD motif binding sites of integrins, are hotspots for MG modification. ${ }^{16}$ Binding of PDLFs to collagen-I is profoundly weakened by MG modification, decreasing binding affinity by ca. 100 -fold. ${ }^{1617}$
MG is mainly metabolized by glyoxalase 1 (Glo1) of the cellular glyoxalase system. Glo1 catalyses the reduced glutathione (GSH)-dependent metabolism of MG to S-D-lactoylglutathione (SLG). The second enzyme of the glyoxalase pathway, glyoxalase 2 (Glo2), catalyses the hydrolysis of SLG to D-lactate, reforming GSH consumed in the Glo1-catalyzed step (figure 1B). There is often limited onward metabolism of D-lactate. In such instances, the flux of formation of D-lactate is a surrogate measure for flux of formation of MG. ${ }^{12}$ An effective strategy to counter accumulation of MG is to increase the expression of Glo1. ${ }^{18}$ This may be achieved through a combination of trans-resveratrol and hesperetin (tRESHESP)—also called a 'Glo1 inducer'. ${ }^{19}$ tRES-HESP increases the expression of Glo1 through activation of transcription factor, nuclear factor erythroid 2-related factor 2 (Nrf2) and binding of Nrf2 to a functional antioxidant response element (ARE) in the GLO1 gene. ${ }^{18}$

An experimental model of PDF dysfunction in diabetes is incubation of PDLFs in primary culture with high glucose (HG) concentration. ${ }^{20}{ }^{21}$ Typically, PDLFs divide and proliferate to confluence over $3-4$ days. ${ }^{20}$ Herein, we propose and test the hypothesis that PDLFs incubated in HG concentration develop dicarbonyl stress associated with HK2-linked glycolytic overload and MG-mediated weakened attachment to collagen-I. We also explore if this may be prevented by treatment with tRES-HESP.

\section{RESEARCH DESIGN AND METHODS}

\section{Materials}

Primary human PDLFs (cat. no. 2630) and Modified Eagles Medium (MEM) (cat. no. M8042) were purchased from ScienCell (Carlsbad, USA). L-Glutamine (200 mM solution; cat. no. 25 030-024) and trypsin/EDTA (cat. no. 25 200-072) were purchased from Invitrogen Life Technologies (Paisley, UK). Tissue culture grade plastic polystyrene T75 flasks was purchased from Fisher Scientific (Nunclone, Loughborough, UK). Aminoguanidine (AG) hydrochloride, 5,5'-dithiobis (2-nitrobenzoic acid) (DTNB), fetal bovine serum (FBS; cat. no. F7524), D-glucose, GSH, oxidized glutathione (GSSG), [glycine$\left.{ }^{13} \mathrm{C}_{2},{ }^{15} \mathrm{~N}\right] \mathrm{GSH}\left(98 \%{ }^{15} \mathrm{~N}\right.$ and $\left.99 \%{ }^{13} \mathrm{C}\right)$, HESP, D-lactate, L-lactate, D-lactic dehydrogenase (cat. no. L9636), L-lactic dehydrogenase (cat. no. L2625), leucine aminopeptidase (cat. no. L6007), pepsin (cat. no. 516360), prolidase (cat. no. P6675), pronase E (cat. no. P5147), penicillinstreptomycin (cat. no. P0781), tRES, trypan blue dye was from Sigma-Aldrich. $\left[{ }^{13} \mathrm{C}_{4}^{15} \mathrm{~N}_{2}\right]$ GSSG was synthesized in-house from [ glycine $\left.-{ }^{13} \mathrm{C}_{2},{ }^{15} \mathrm{~N}\right] \mathrm{GSH}$ as described. ${ }^{19} \mathrm{MG}$ for cell culture was prepared and purified as previously described. ${ }^{22}$ Methanol, acetonitrile and tetrahydrofuran (all HPLC grade) were purchased from Fisher Scientific. Trifluoroacetic acid ( $\geq 99.0 \%$ HPLC grade) and trichloroacetic acid (BioUltra, $\geq 99.5 \%$ ) were purchased from Sigma-Aldrich. All other reagents, salts, acids and bases for buffers were analytical grade reagents and purchased from Sigma-Aldrich and Fisher Scientific. 
A
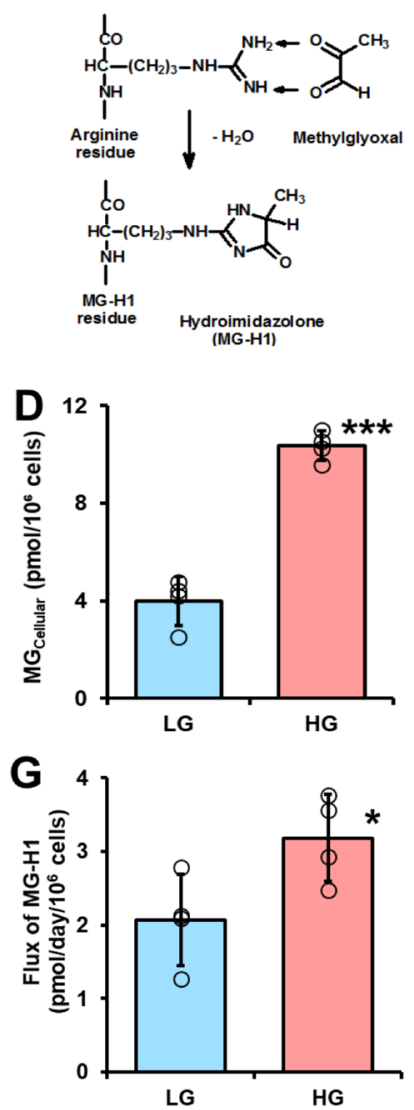

B
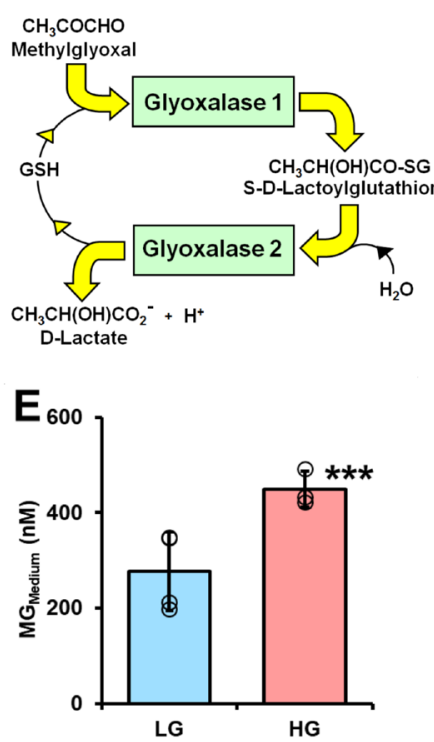

H

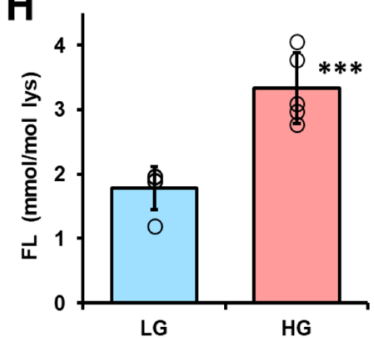

C
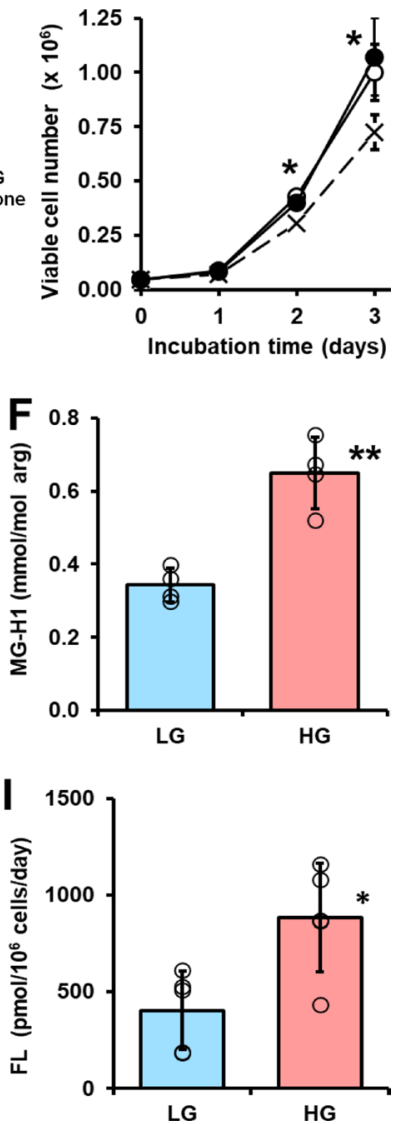

Figure 1 Dicarbonyl stress in human periodontal ligament cells in high glucose concentration cultures in vitro. (A) Protein glycation by methylglyoxal (MG) forming arginine-derived MG-H1 adduct. (B) Metabolism of MG by the glyoxalase system. (C-I) Culture of periodontal ligament fibroblasts (PDLFs) for 3 days and effect of glucose concentration. (C) Viable cell number and effect of initial glucose concentration. Key: $x--x, 5 \mathrm{mM}$ glucose; $\circ-\circ, 8 \mathrm{mM}$ glucose and $\bullet-\bullet, 25 \mathrm{mM}$ glucose. (D) Cellular $M G$ content $(n=4)$. (E) MG concentration of the culture medium $(n=4)$. (F) MG-H1 content of cell protein. (G) Flux of release of MG-H1 free adduct into the culture medium. (H) FL content of cell protein. (I) Flux of release of FL free adduct into the culture medium. Data are mean $\pm S D(C) n=3$; (D-G) $n=4$; (H and I) $n=5)$. ${ }^{*}<<0.05 ;{ }^{* *} p<0.01 ;{ }^{* *} p<0.001$; Student's $t$-test. FL, $N_{\varepsilon}$-fructosyllysine; HG, high glucose; LG, low glucose.

\section{Culture of human periodontal ligament fibroblasts}

Human PDLFs were cultured in MEM supplemented with 2 mM L-alanyl-L-glutamine, 10\% FBS, 100 units/ $\mathrm{mL}$ penicillin, $100 \mu \mathrm{g} / \mathrm{mL}$ streptomycin at $37^{\circ} \mathrm{C}$ under aseptic conditions and $5 \%$ carbon dioxide/air with $100 \%$ humidity. PDLFs were passaged every 3 days and with a seeding density of ca. 5000 cells $/ \mathrm{cm}^{2}$. For experiments performed in low glucose (LG) and high glucose (HG) conditions, culture media was supplemented with 8 and $25 \mathrm{mM}$ glucose, respectively, for 3 days (unless otherwise stated) using cells between passages 3 and 5 . Cell viability was assessed by the Trypan blue exclusion method. For metabolic flux measurements, analytes were determined at baseline and day 3 and the mean rate of change deduced. The effect of Glo1 inducer, tRES-HESP (10 $\mu \mathrm{M})$, on metabolic dysfunction of PDLFs in HG concentration cultures was investigated.

\section{Biochemical measurements}

Glucose concentration was assayed by the hexokinase method. L-lactate and D-lactate concentrations were assayed by end point enzymatic assay. Activities of Glo1, MG reductase and MG dehydrogenase were assayed as described. ${ }^{23}$ The following analytes were determined by stable isotopic dilution analysis liquid chromatographytandem mass spectrometry (LC-MS/MS): MG content of PDLFs and culture medium ${ }^{24}$; MG-H1, glucose-derived glycation adduct, $\mathrm{N}_{\varepsilon}$-fructosyl-lysine (FL) and oxidative damage markers, methionine sulfoxide (MetSO), dityrosine, N-formylkynurenine (NFK) and 3-nitrotyrosine (3-NT) in cytosolic protein extracts and related free adducts in culture medium ${ }^{25}$ and the cellular contents of GSH, GSSG and SLG. ${ }^{19}$ Cellular protein thiols were deduced by determining total cellular thiols, derivatized with $\mathrm{DTNB}^{26}$ and subtracting the cellular content of GSH. For assay of determining total cellular thiols, PDLFs $\left(1 \times 10^{6} \mathrm{cells}\right)$ were sonicated $(110 \mathrm{~W}, 30 \mathrm{~s})$ on ice in $10 \mathrm{mM}$ sodium phosphate buffer, $\mathrm{pH}$ 7.0. Cell membranes were sedimented by centrifugation (20 $000 \mathrm{~g}, 30 \mathrm{~min}, 4^{\circ} \mathrm{C}$ ) and the supernatant cell extract retained. DTNB solution $(125 \mu \mathrm{L}, 1 \mathrm{mM}$ DTNB in 100 
$\mathrm{mM}$ sodium phosphate buffer, $\mathrm{pH} 7.4$, with $0.2 \mathrm{mM}$ DETAPAC (diethylenetriaminepenta-acetic acid)) was added to water $(100 \mu \mathrm{L})$ and cell extract $(25 \mu \mathrm{L})$. The absorbance increase at $405 \mathrm{~nm}$ after $20 \mathrm{~min}$ was recorded and used to deduce thiol content, calibrated by assay of 0-30 nmol GSH. Real-time PCR and Western blot analysis was performed as previously described, ${ }^{12} 18$ normalized to housekeeping protein, $\beta$-actin.

\section{Proteomics and bioinformatics analysis}

Cytosolic protein extracts were analyzed by highresolution Orbitrap mass spectrometry of tryptic digests, as described. ${ }^{27}$ Protein extracts were prepared from PDLFs incubated for 3 days with LG and HG concentration; and also cytosolic protein extract incubated with $500 \mu \mathrm{M}$ MG for 24 hours at $37^{\circ} \mathrm{C}$, increasing the MG-H1 residue content by 10 -fold. Reduced and alkylated protein extracts were digested with Lys-C and TPCKtreated trypsin. Mean sequence coverage for proteins identified in PDLF extracts was 16.7\%. Label-free quantitation of protein abundances were determined in three independent biological replicate samples using Progenesis QI for proteomics 2.0 software (Nonlinear Dynamics, Newcastle, UK). Protein ontology was evaluated using the Database for Annotation, Visualization and Integrated Discovery V.6.8 (https://david.ncifcrf.gov/). ${ }^{28}$ REACTOME analysis ${ }^{29}$ was used for pathway enrichment analysis. MG modification occurring in protein functional domains was identified by receptor binding domain (RBD) analysis, as described. ${ }^{12} 29$

\section{Statistical analysis}

Data are mean \pm SD of $\geq 3$ independent biological replicates and responses were validated for PDLFs from three different donors. Test and control samples were analyzed randomly. Significance of difference of two groups by Student's t-test and of $>2$ groups by one-way analysis of variance.

\section{RESULTS}

\section{Effect of glucose concentration on growth and viability of} human periodontal ligament fibroblasts in vitro

Initial investigations of the effect of glucose concentration on the growth of PDLFs in primary culture revealed there was similar rapid growth of PDLFs in culture medium containing 8 and $25 \mathrm{mM}$ glucose over 3 days whereas there was partial growth arrest without decrease in viability in medium containing $5.5 \mathrm{mM}$ glucose (figure 1C). In the latter case, the final glucose concentration was ca. $3 \mathrm{mM}$-typical of hypoglycemia. In subsequent experiments, we employed culture medium containing 8 and $25 \mathrm{mM}$ glucose as models of normoglycemia or LG concentration conditions and hyperglycemia or HG concentration conditions. In support of this, the mean salivary glucose concentration in healthy human subjects is ca. 7-8 $\mathrm{mM}$ and increases to over $23-25$ $\mathrm{mM}$ in patients with diabetes. ${ }^{30}$ PDLFs cultured under these conditions gave reproducible cell growth kinetics over the passage range studied. The population doubling time was ca. 0.6 days in medium containing 8 and $25 \mathrm{mM}$ glucose. There was no decrease in cell viability under these culture conditions.

\section{Dicarbonyl stress in human periodontal ligament fibroblasts} in high glucose concentration cultures in vitro

When PDLFs were incubated in HG concentration for 3 days there was an increase in cellular concentration of MG, compared with LG concentration control: $10.36 \pm 0.60$ vs $3.98 \pm 1.00 \mathrm{pmol} / 10^{6}$ cells, $\mathrm{p}<0.001$ (figure 1D). PDLF cell volume is ca. $2.5 \mathrm{pL}$ per cell, ${ }^{31}$ indicating that the concentration of MG in PDLFs is ca. 1.6 $\mu \mathrm{M}$ in LG concentration, increased to $4.2 \mu \mathrm{M}$ in HG concentration. This is keeping with the concentration of MG in other human cell types of $2-4 \mu \mathrm{M} .^{24}$ Incubation of PDLFs with HG concentration also produced an increased MG concentration in the culture medium: $449 \pm 38 \mathrm{nM}$ vs $279 \pm 82 \mathrm{nM}$, $\mathrm{p}<0.05$ (figure $1 \mathrm{E}$ ). This increased cellular concentration of MG was associated with an increased steady-state level of MG-H1-modified cellular protein and increased flux of excretion of MG-H1 free adduct in the culture mediumthe latter formed mainly by proteolysis of MG-H1modified cellular protein (figure $1 \mathrm{~F}$ and G). There was a similar increase in glucose-derived glycation adduct, FL, content of cell protein-reflecting increased cellular glucose concentration and increased excretion of related FL free adduct (figure $1 \mathrm{H}$ and I). The cellular protein content of oxidative damage markers was unchanged in HG concentration: MetSO, $0.89 \pm 0.07$ vs $1.14 \pm 0.43 \mathrm{~mol} /$ mol met; dityrosine $0.080 \pm 0.055$ vs $0.058 \pm 0.031 \mathrm{mmol} /$ mol tyr; NFK, $0.109 \pm 0.042$ vs $0.091 \pm 0.030 \mathrm{mmol} / \mathrm{mol}$ trp and 3-NT, $0.0030 \pm 0.0013$ vs $0.0019 \pm 0.0009 \mathrm{mmol} /$ mol tyr. We also measured the cellular contents of GSH, GSSG and protein thiols. The cellular contents of GSH, GSSG and total GSH were decreased by $25 \%$ in PDLFs incubated in HG concentration. Decreased cellular GSH concentration is expected to contribute to increased MG concentration of PDLFs in HG concentration conditions by decreasing in situ activity of Glo1. ${ }^{13}$ Surprisingly, there was a $25 \%$ increase of cell protein thiols and $13 \%$ increase in total cellular thiols (GSH+protein thiols) in the HG concentration culture (figure 2A-2E).

PDLFs were relatively resistant to toxicity induced by exogenous MG: incubation of PLDFs with 25-1000 $\mu \mathrm{M}$ for 2 days produced growth arrest and toxicity at $\geq 200$ $\mu \mathrm{M} M G-a$ concentration profoundly greater than physiological extracellular concentrations of MG. The median growth inhibitory concentration $\mathrm{GC}_{50}$ of $\mathrm{MG}$ was $250 \pm 3$ $\mu \mathrm{M}(\mathrm{n}=18)$.

A metabolic driver of increased cellular concentration of MG in the HG concentration culture is increased formation of MG, as judged by increase in formation of D-lactate-a surrogate indicator of flux of formation of $\mathrm{MG}^{12}$ (figure 2F). The flux of D-lactate in PDLFs in LG concentration was ca. $6 \mathrm{nmol} / 10^{6}$ cells/day, equivalent to ca. $0.06 \%$ flux of glucotriose. The increase in flux of formation of D-lactate was concomitant with increased 

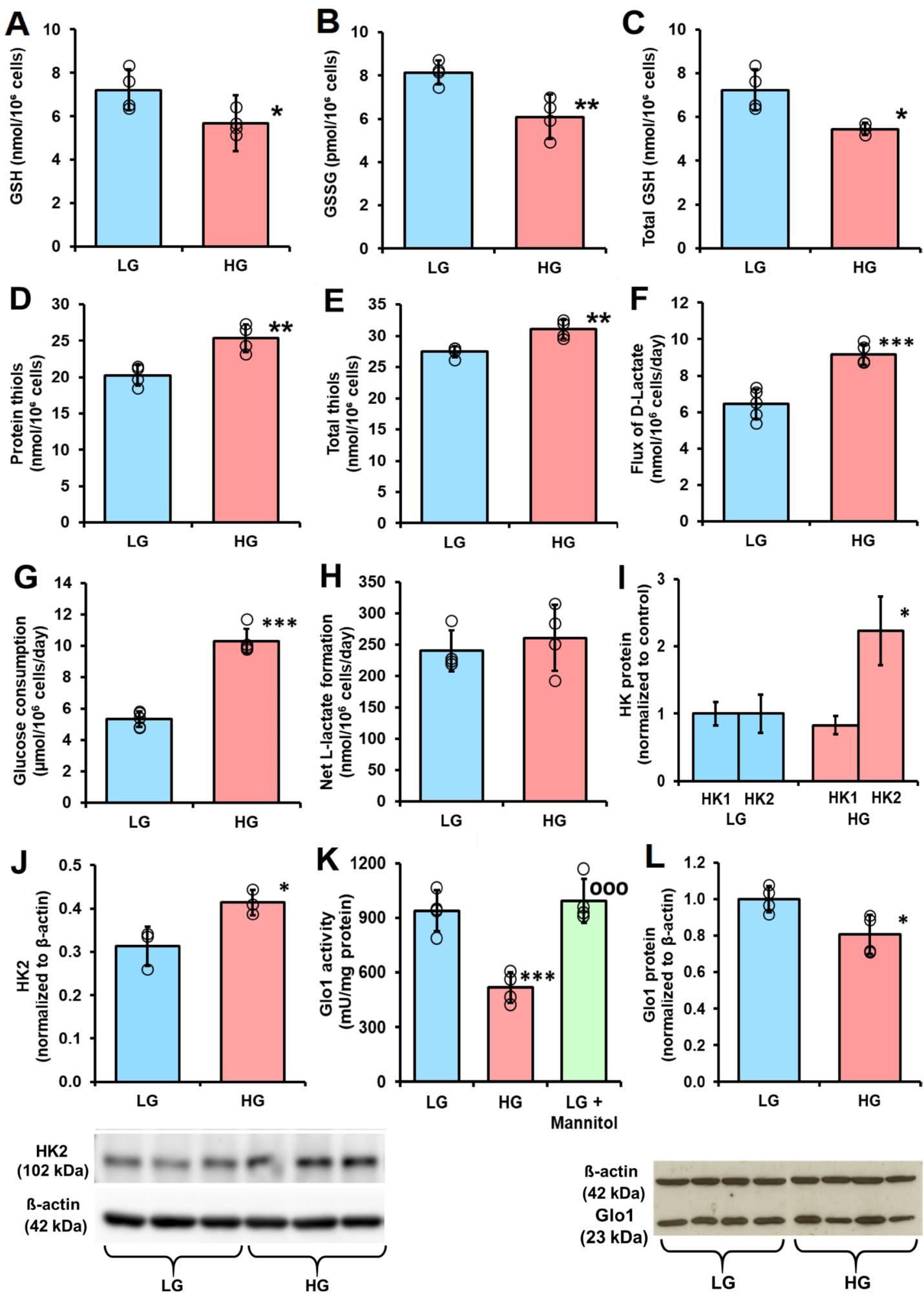

Figure 2 Cellular glutathione and protein thiol status and metabolic drivers of dicarbonyl stress in human periodontal ligament cells in high glucose concentration cultures in vitro. (A), (B) and C), Cellular glutathione (GSH), oxidized glutathione (GSSG) and total GSH, respectively. (D) Cell protein thiols. (E) Total cell thiols. (F) Flux of formation of D-lactate. (G) Glucose consumption. $(\mathrm{H})$ Net formate of L-lactate. (I) Relative abundance of hexokinase isozyme proteins in periodontal ligament fibroblasts (PDLFs) determined by label-free, high mass resolution Orbitrap proteomics. (J) Hexokinase-2 protein abundance by western blot analysis (gel image used in densitometric quantitation is given below). (K) Activity of glyoxalase 1 (Glo1). (L) Glo1 protein abundance by western blot analysis (gel image used in densitometric quantitation is given below). Data are mean $\pm \mathrm{SD}(\mathrm{A}-\mathrm{H})$, $(\mathrm{K})$ and $(\mathrm{L}), \mathrm{n}=4$; (I) and $(\mathrm{J}), \mathrm{n}=3 .{ }^{*} \mathrm{P}<0.05 ;{ }^{* *} \mathrm{p}<0.01 ;{ }^{* \star *} \mathrm{p}<0.001$; Student's $t$-test. HG, high glucose; HK, hexokinase; LG, low glucose. 
flux of glucose consumption by PDLFs (figure 2G). There was no net increase in formation of L-lactate (figure $2 \mathrm{H}$ ). The flux of D-lactate formation expressed as a proportion of flux of glucotriose metabolism ( $2 \times$ glucose consumption) was ca. $0.06 \% \pm 0.01 \%$ and was not increased in $\mathrm{HG}$ concentration. Seeking evidence for an explanation for increased glucose consumption in HG conditions, we analyzed abundances of hexokinases of PDLFs, hexokinase-1 (HK1) and HK2 by quantitative proteomics. The relative abundance of HK1 was unchanged in HG conditions whereas the relative abundance of HK2 increased twofold (figure 2I). This was corroborated by Western blot analysis with quantitative densitometry (figure 2J).

A further factor influential on the cellular concentration of MG are activities of enzymes of MG metabolism: Glo1, MG reductase and MG dehydrogenase. The activity of Glo1 in PDLFs in LG concentration cultures was $985 \pm 148 \mathrm{mU} / \mathrm{mg}$ protein. This is intermediate between that of human aortal endothelial cells (HAECs) $(1862 \pm 178 \mathrm{mU} / \mathrm{mg}$ protein) and red blood cells (RBCs) $\left(110 \pm 14 \mathrm{mU} / \mathrm{mg}\right.$ protein). ${ }^{12}{ }^{32}$ Glo1 was the major pathway of MG metabolism as activities of other MG metabolizing enzymes were much lower than of Glo1: the activity of MG reductase was $0.09 \pm 0.02$ units $/ \mathrm{mg}$ protein and was not changed in HG concentration cultures, and the activity of MG dehydrogenase was below the limit of detection $(<0.0005 \mathrm{mU} / \mathrm{mg}$ protein $)$ in all culture conditions. The activity of Glo1 was decreased $45 \%$ in HG concentration cultures, with respect to LG concentration (figure 2K). A similar decrease was not produced when PDLFs were incubated with LG concentration and $17 \mathrm{mM}$ mannitol, indicating the HG response was not produced by increased osmolarity (figure $2 \mathrm{~K}$ ). There was no decrease of Glo1 mRNA in HG concentration cultures (ratio of Glo1/ $\beta$-actin mRNA: LG, $0.792 \pm 0.002$, $\mathrm{HG}, 0.791 \pm 0.003)$ but there was with a similar decrease in Glo1 protein (figure 2L). We studied the half-life of Glo1 protein by following the decrease of Glo1 protein following block of Glo1 synthesis with cycloheximide. The half-life of Glo1 was decreased in cultures with HG concentration, with respect to LG concentration control $(1.31 \pm 0.15$ vs $2.30 \pm 0.37$ days, $\mathrm{n}=3, \mathrm{p}<0.01)$. Therefore, in HG concentration cultures, Glo1 activity and protein are decreased by increased proteolysis.

The activity of Glo2 in PDLFs in LG concentration was $2.22 \pm 0.65 \mathrm{mU}$ per $\mathrm{mg}$ protein; $\mathrm{cf}$. $64 \mathrm{mU} / \mathrm{mg}$ protein in RBCs. ${ }^{32}$ The relative activities of Glo1 and Glo2 in situ with physiological concentrations of substrate, deduced applying related $\mathrm{K}_{\mathrm{M}}$ and $\mathrm{k}_{\text {cat }}$ values, are such that the cellular concentration of SLG is very low-usually $<0.1 \%$ of total cell GSH. ${ }^{33}$ This was the case for PDLFs where the concentration of SLG was below the limit of detection $\left(<1.1 \mathrm{pmol} / 10^{6}\right.$ cells $)$.

\section{Cytosolic proteome of human periodontal ligand fibroblasts in} high glucose concentration cultures in vitro

We detected and quantified proteins in cytosolic extracts of PDLFs by high-resolution Orbitrap mass spectrometry proteomics. A total of 1105 cytosolic protein were identified and quantified in PDLFs. The abundance of 144 proteins was increased in HG concentration conditions (online supplemental table S1). The five proteins of highest abundance change were: ATPbinding cassette subfamily E member 1, +9-fold; Axin interactor, dorsalization-associated protein, +8-fold; E3 ubiquitin-protein ligase HUWE1, +6-fold; isoform 2 of electron transfer flavoprotein subunit beta, +6 -fold and HK2, +3-fold. Pathways enrichment analysis of protein abundance changes showed increase in chaperonin folding pathway of chaperonin containing T-complex protein 1 (CCT) - also known as T-complex protein-1 Ring Complex (TRiC), heat shock protein 90 (HSP90) chaperone cycle for steroid hormone receptors, Golgito-endoplasmic reticulum (ER) retrograde traffic and cellular responses to stress, including response to heat stress and glucose metabolism. Mean abundance changes were increases of $49 \%-61 \%$ (table 1 ). Only one protein was of decreased abundance: platelet-activating factor acetyl-hydrolase IB subunit alpha-an initiation factor for dynein-driven organelle transport along microtubules; $-24 \%$.

To gain insight into which proteins have endogenous MG modification, we interrogated proteomics data for evidence of proteins with MG-H1 (+54.01 Da mass increment on arginine residues). MG-H1 modification was detected on five proteins (with arginine modification site): $\beta$-actin (R116), $\gamma$-actin (R206), filamin-A (R1959), glutamine-rich protein-1 (R669) and U6 Sm-like protein LSm3 (R80). MG modification of $\beta$-actin and U6 Sm-like protein LSm3 (LSM3) was in a predicted functional domain and thereby likely produces functional impairment. Further proteins at risk of modification by MG were identified by incubating cytosolic protein with exogenous MG to increase mean modification by MG-H1 10-foldthe maximum upper limit of the MG increase in blood samples of patients with diabetes. ${ }^{32}$ We then detected MG-H1 modification on 172 proteins in 353 unique modification sites (online supplemental table S2). An example of detection of MG-H1 modification is given for modified R206 on $\gamma$-actin (figure 3A) with the related RBD plot (figure 3B). Pathways analysis showed that MG modification was enriched in protein processing in ERincluding modification of several HSPs, carbon metabolism-including glycolytic, pentosephosphate pathway and mitochondrial enzymes (online supplemental table S3). From RBD analysis, 115 of the 353 (33\%) modifications were in predicted function domains and 74 of the 172 proteins $(43 \%)$ had one or more MG modifications in a functional domain.

\section{Prevention of dicarbonyl stress and dysfunction of human periodontal ligament fibroblasts in high glucose concentration by Gllo1 inducer}

In previous studies, tRES-HESP corrected dicarbonyl stress in HEACs in HG concentration ${ }^{12}$ and dicarbonyl stress of overweight and obese human subjects in a 
Table 1 Pathways enrichment analysis of proteins increased in high glucose cultures

\begin{tabular}{|c|c|c|c|c|}
\hline Pathway name & Count & P value* & $\begin{array}{l}\text { Abundance } \\
\text { ratio } \\
\text { Mean+SD }\end{array}$ & Proteins (Uniprot ID) \\
\hline Folding of actin by CCT/TRiC & 5 & 1.37E-06 & $1.61 \pm 0.17$ & TCPA, TCPB, TCPD, TCPH, TCPZ \\
\hline $\begin{array}{l}\text { Cooperation of prefoldin and } \\
\text { TRiC/CCT in actin and tubulin } \\
\text { folding }\end{array}$ & 6 & $3.50 \mathrm{E}-05$ & $1.60 \pm 0.15$ & PFD4, TCPA, TCPB, TCPD, TCPH, TCPZ \\
\hline $\begin{array}{l}\text { HSP90 chaperone cycle for } \\
\text { steroid hormone receptors }\end{array}$ & 7 & 8.54E-05 & $1.53 \pm 0.08$ & $\begin{array}{l}\text { CAZA1, CAZA2, DC112, DC1L2, DCTN2, DNJA2, } \\
\text { HS71A, }\end{array}$ \\
\hline $\begin{array}{l}\text { COPI-independent Golgi-to- } \\
\text { ER retrograde traffic }\end{array}$ & 6 & 4.85E-04 & $1.50 \pm 0.10$ & CAZA1, CAZA2, DC1I2, DC1L2, DCTN2, RBGPR \\
\hline $\begin{array}{l}\text { Golgi-to-ER retrograde } \\
\text { transport }\end{array}$ & 9 & $8.43 \mathrm{E}-04$ & $1.58 \pm 0.21$ & $\begin{array}{l}\text { ARF4, CAZA1, CAZA2, DCL1I2, DCL1L2, DCTN2, } \\
\text { GBF1, KINH, RBGPR. }\end{array}$ \\
\hline Cellular responses to stress & 17 & 2.09E-03 & $1.54 \pm 0.13$ & $\begin{array}{l}\text { CAZA1, CAZA2, DC112, DC1L2, DCTN2, DNAJ2, } \\
\text { GBF1, HS71A, PRDX2, MK03, NU214, PSDE, } \\
\text { PSMD1, PSMD3, SEC13, TERA, TPR }\end{array}$ \\
\hline $\begin{array}{l}\text { Cellular response to heat } \\
\text { stress }\end{array}$ & 7 & $2.02 \mathrm{E}-03$ & $1.57 \pm 0.16$ & GBF1, HS71A, HS105, MK03, SEC13, TERA, TPR. \\
\hline Glucose metabolism & 7 & $2.43 \mathrm{E}-03$ & $1.49 \pm 0.11$ & 2AAA, AATC, GNPI2, NU214, PFKAP, SEC13, TPR \\
\hline $\begin{array}{l}\text { Intra-Golgi and retrograde } \\
\text { Golgi-to-ER traffic }\end{array}$ & 10 & 4.33E-03 & $1.61 \pm 0.24$ & $\begin{array}{l}\text { ARF4, CAZA1, CAZA2, DC1I2, DC1L2, DCTN2, } \\
\text { GBF1, GOGA4, KINH, RBGPR, }\end{array}$ \\
\hline $\begin{array}{l}\text { COPI-mediated anterograde } \\
\text { transport }\end{array}$ & 7 & $3.08 \mathrm{E}-03$ & $1.61 \pm 0.23$ & $\begin{array}{l}\text { ARF4, CAZA1, CAZA2, DC1I2, DC1L2, DCTN2, } \\
\text { GBF1 }\end{array}$ \\
\hline
\end{tabular}

${ }^{*} \mathrm{P}$ value is Bonferroni corrected and false discovery rate is $<0.05$.

CCT, chaperonin containing T-complex protein 1; COPI, coat protein complex I; ER, endoplasmic reticulum; HSP, heat shock protein; TRiC,

T-complex protein-1 ring complex.

double-blind, randomized placebo-controlled clinical trial. ${ }^{19}$ Incubation of PDLFs with $10 \mu \mathrm{M}$ tRES-HESP for 3 days increased Glo1 activity of PDLFs in cultures with LG concentration and prevented the decrease of Glo1 activity of PDLFs in cultures with HG concentration (figure 4A). This was associated with the correction of increased cellular MG concentration in HG concentration cultures (figure 4B). Remarkably, treatment with tRES-HESP produced a small decrease in flux of formation of D-lactate in LG concentration cultures and correction of increased flux of formation of D-lactate in HG concentration cultures, suggesting that the flux of formation of MG was decreased in both conditions (figure 4C). A similar effect was found for glucose consumption (figure 4D), indicating that flux of formation of MG was decreased by limiting glucose metabolism in LG concentration cultures and correcting increased glucose consumption in HG concentration cultures. There was an associated correction of increased steadystate level of MG-modified cell protein and increased flux of protein modification by MG in HG concentration cultures by treatment with tRES-HESP (figure $4 \mathrm{E}$ and F). Treatment of PDLFs with tRES-HESP also increased the cellular concentration of GSH in LG and HG concentration cultures (figure 4G) and increased the attachment of PDLFs to collagen-I in LG and HG concentration (figure $4 \mathrm{H}$ ). Decreased attachment to collagen-I of PDLFs incubated in HG concentration was also prevented by incubation with the MG scavenging agent, $(500 \mu \mathrm{M}),{ }^{34}$ as judged by absorbance increase reflecting light scattering of collagen-I sequestered cells (absorbance at 595 nm: LG, 0.062 $\pm 0.008 ; \mathrm{LG}+\mathrm{AG}, 0.081 \pm 0.005, \mathrm{p}<0.05$; HG, $0.035 \pm 0.003, \mathrm{p}<0.01$ and $\mathrm{HG}+\mathrm{AG}, 0.082 \pm 0.003, \mathrm{p}<0.05$ and $\left.\mathrm{p}^{\prime}<0.001\right)$.

\section{DISCUSSION}

Herein, we found HG concentration imposed dicarbonyl stress on human PDLFs in vitro. The cellular concentration of MG was increased, leading to increased MG modification and related misfolding of cell protein, a proteomic response to cell stress and weakened binding of PDLFs to collagen-I. Dicarbonyl stress and these related responses were prevented by treatment of PDLF cultures with tRES-HESP.

PDLFs express glucose transporters, GLUT1, GLUT3 and GLUT4 $4{ }^{35}$ In HG concentration cultures, there is increased uptake of glucose by GLUT1 and GLUT3 and increased cytoplasmic glucose concentration. The latter was evidenced by the ca. twofold increase in the steadystate level of FL residue adduct content of cell protein found herein (figure 1H). PDLFs express HK1 and HK2 which limit the rate of glucose metabolism; both hexokinases are operating under glucose saturation kinetics in 
A

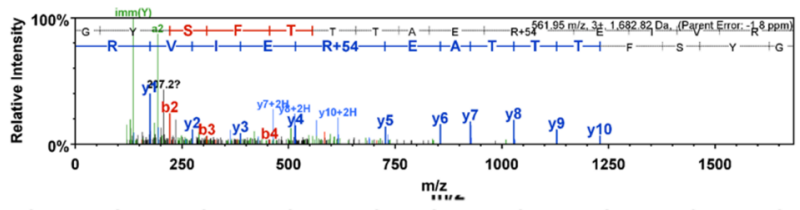

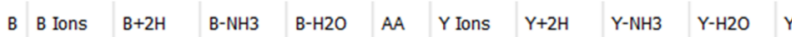

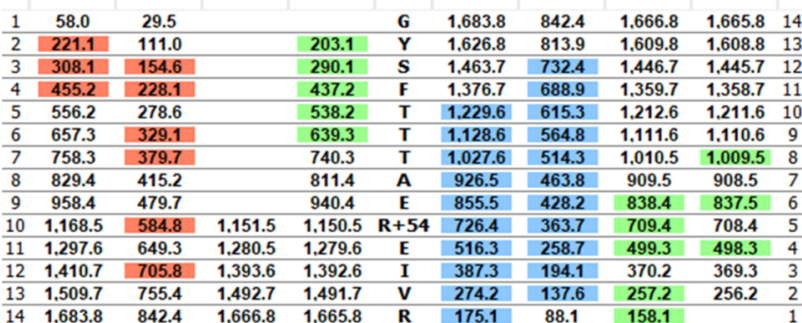

B

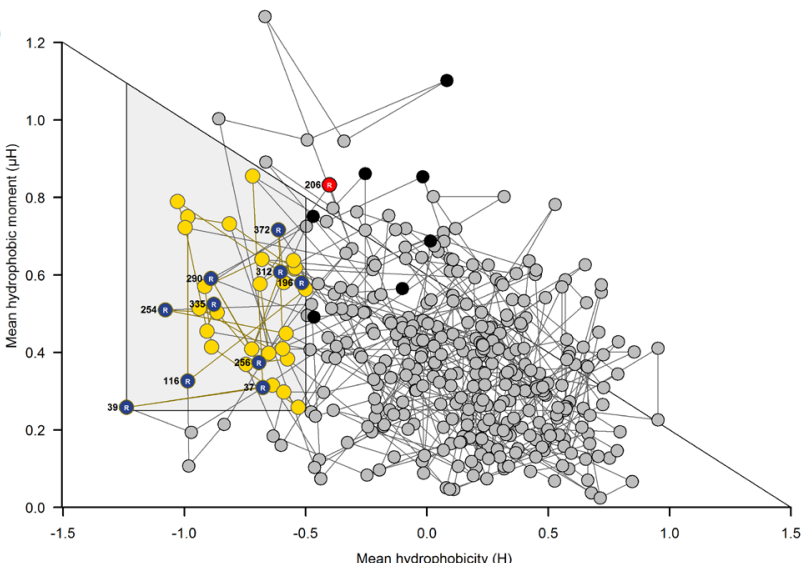

Figure 3 Mass spectrometric detection of methylglyoxal (MG)-modified proteins and prediction of functional impact by receptor binding domain (RBD) analysis. (A) MG-modified peptide identification. $\gamma$-Actin, R206. Peptide fragmentation ion mass scan of peptide 197-210 with MG-H modification on R206 (+54.01): GYSFTTTAER ${ }_{\text {GG-H1 }}$ EIVR; with fragment ion assignment table. lons detected are color coded in the mass spectrum assignments with color shading in the table. $\mathrm{m} / \mathrm{z}$ ion values unshaded were not detected and black spaces in the table indicate ions are not expected chemically. Unique peptide with: peptide identity probability 98.6 , mascot ion score 55.3, observed mass (m/z) $561.95(3+)$, actual peptide mass $1682.82 \mathrm{amu}$, calculated +1 hour peptide mass $1683.83 \mathrm{amu}$. 'Parent mass error' indicates error on the peptide mass. (B) RBD plot for human $\gamma$-actin. Line-linked filled circles represent the primary sequence. The RBD is the area bound by the trapezium in the upper left-side region of the chart. Key: blue and red filled circles, MG-H1 residue inside (R196, R312, R372) and outside (R206) the RBD, respectively; black filled circle, unmodified arginine residues in the RBD; yellow-filled circle, other amino acid residues in the RBD other amino acid residues in the RBD and gray filled circle, amino acid residues outside the RBD.

both LG and HG concentration conditions. ${ }^{36}$ Increased glucose consumption by fibroblasts was observed herein, with onward metabolism through glycolysis and tricarboxylic acid cycle. ${ }^{37}$ This occurs by HK2-linked glycolytic overload. ${ }^{38}$ HK2, unlike HK1, is degraded by chaperonemediated autophagy through motif $_{712}$ QRFEK $_{716}$. This motif, in the glucose binding site of the C-terminal domain, is masked in HG concentration, stabilizing HK2 to proteolysis. ${ }^{12}{ }^{39}$ Herein, we found HK2 but not HK1 protein of PDLFs increased in HG concentration (figure 2I and online supplemental table S1). The flux of glucose metabolism is very sensitive to change in level of HK2 protein, compared with change in level of HK1 protein, because the high turnover number $\mathrm{k}_{\text {cat }}$ of HK2; $\mathrm{k}_{\text {cat,HK2 }} / \mathrm{k}_{\text {cat,HK1 }} \approx 5{ }^{36}$ Increase of HK2 protein explains the overall increase in glucose consumption by PDLFs in cultures with HG concentration; cf. HAECs cultured in HG concentration. ${ }^{12}$ Increased glucose metabolism by HK2 produces increased flux through glycolysis without increased abundance of other glycolytic enzymes, except for ATP-dependent 6-phosphofructokinase (PFKAP) (table 1)—conditions for glycolytic overload. ${ }^{11}$ Steadystate concentrations of triosephosphate glycolytic intermediates are thereby increased proportionate to increase in glycolytic flux, with consequent increase in formation of MG. Increased flux of MG formation-as indicated by increased flux of D-lactate, decreased of cellular GSH and decreased activity of Glo1 synergize to increase the cellular concentration of MG in HG concentration.

The concentration of GSH and GSSG in PDLFs was ca. 7 and $0.012 \mathrm{nmol}$ per $10^{6}$ cells, respectively, under LG concentration conditions, indicating $0.3 \% \mathrm{GSH}$ is oxidized, that is, PDLFs have a strongly reduced cytosol. The GSH estimate is similar to that found previously for PDLFs. ${ }^{40}$ The GSH/GSSG ratio did not change in HG concentration but the cellular concentration of both GSH and GSSG were decreased by $25 \%$. This suggests GSH synthesis of PDLFs may be modestly impaired in HG concentration cultures. In contrast, the cellular concentration of protein thiols (cysteinyl thiols) was increased. Since cellular GSH concentration was decreased, this is likely due to increased activity of glutaredoxin which is involved in maintaining the reduced status of protein thiols in cells. ${ }^{41}$ Interestingly, increased glutaredoxin was found previously in Muller cells cultured in HG concentration and streptozotocin-diabetic rats, which was linked to activation of inflammatory signaling through cytosolic glutaredoxin-1 induction by the nuclear factor kappa B system. ${ }^{42} 43$

Analysis of the cytosolic proteome showed a remarkable increased protein abundance focused on pathways of chaperonins, cellular responses to stress, including response to heat stress-involving HSPs, and Golgi-to-ER retrograde traffic of proteins. Increased chaperonin TRiC/CCT and HSPs are directly related to the activation of heat shock factor-1 (HSF-1) - part of the UPR. ${ }^{44} 45$ This is likely due to release of HSF-1 from complexation with HSP70 and HSP40 by increased MG-modified misfolded proteins, migration of HSF-1 to the nucleus and increased transcriptional activity for HSPs and chaperonins. ${ }^{12}$ MG-modified proteins are thereby likely funneled through the HSP pathway for degradation by proteasomal proteolysis and chaperone-mediated autophagy. ${ }^{45}$ Interestingly, increased Golgi-to-ER retrograde traffic of proteins is part of the cell response to increased 

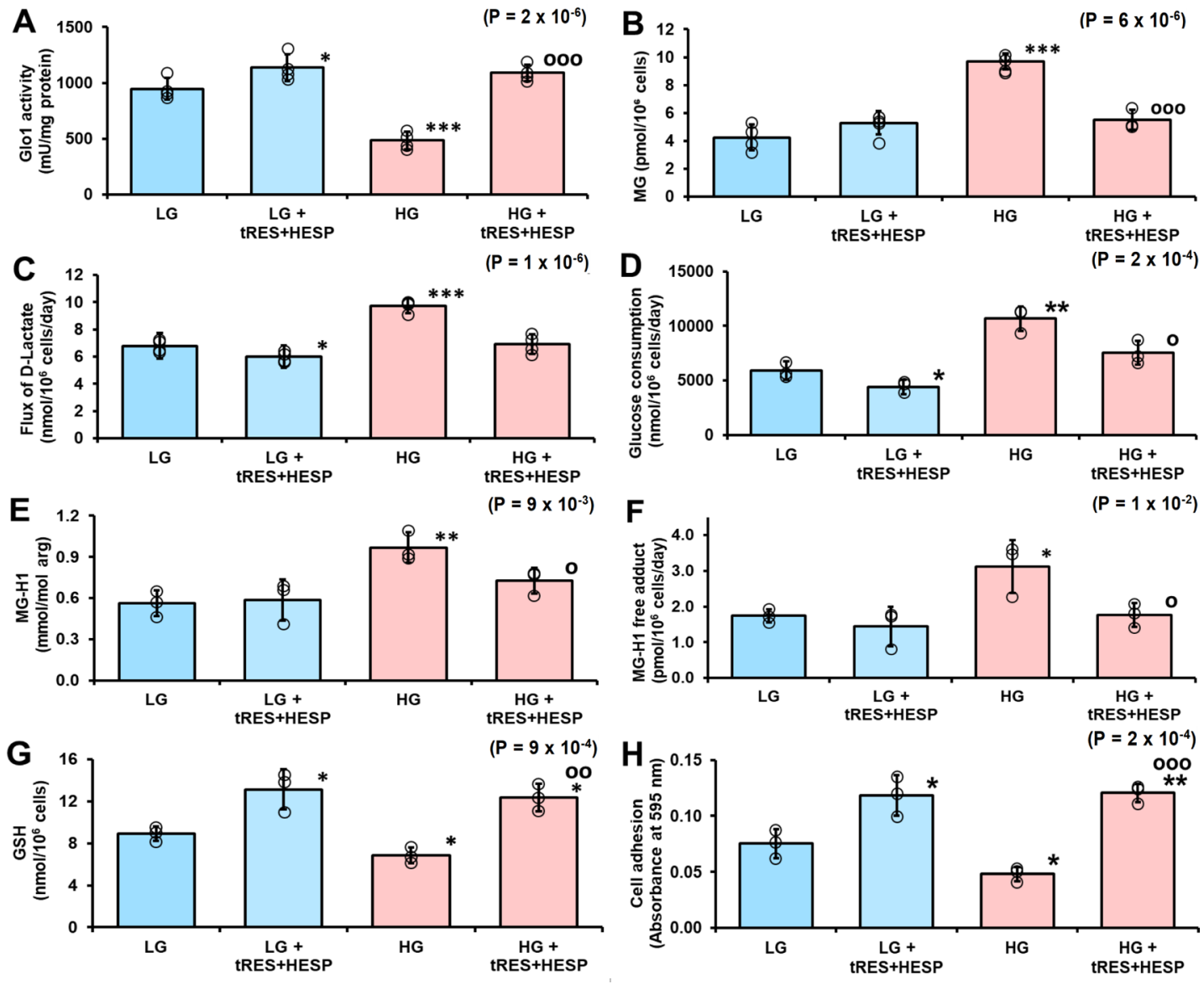

Figure 4 Prevention of dicarbonyl stress and dysfunction of human periodontal ligament fibroblasts in high glucose concentration by glyoxalase 1 (Glo1) inducer. (A) Glo1 activity. (B) Cellular content of methylglyoxal (MG). (C) Flux of formation of D-lactate. (D) Glucose consumption. (E) MG-H1 content of cell protein. (F) Flux of excretion of MG-H1 free adduct. (G) Cellular glutathione (GSH). (H) Binding of periodontal ligament fibroblasts (PDLFs) to collagen-I in vitro. Significance: $p$ value for one-way analysis of variance (ANOVA) is given in the top right-hand corner of each panel. Data are mean $\pm S D(n=4$ except $n=3$ for $D$ ). Significance: $p$ values for one-way ANOVA are given in the top right-hand corner of each panel. ${ }^{*} P<0.05 ;{ }^{* *} p<0.01$; ${ }^{* * *} \mathrm{p}<0.001$ and ${ }^{\circ} \mathrm{p}<0.05 ;{ }^{\circ 0} \mathrm{p}<0.01 ;{ }^{\circ 00} \mathrm{p}<0.001$; Student's t-test. HESP, hesperetin; HG, high glucose; LG, low glucose; transresveratrol.

misfolded proteins, returning misfolded proteins to the ER for refolding. ${ }^{46}$ In the cytosol, misfolded proteins are targeted for degradation by specific ubiquitin-E3-ligases: CHIP (C terminus of heat shock cognate $71 \mathrm{kDa}$ proteininteracting protein) with cognate chaperone HSP90 and E6-AP domain containing protein, HUWE1, with cognate chaperone HSP70. ${ }^{48}$ These proteins had abundance increases of 2-fold, 2.5-fold, 5.6-fold and 1.6-fold, respectively, in HG cultures. HUWE1 targets proteins with 'unshielded' surface hydrophobic regions which the arginine to MG-H1 modification produces, consistent with increased MG modified and misfolded proteins being targeted for degradation. Interestingly, there is a suggestion from bioinformatics protein-protein interaction studies aggregated in the Harmonizome database that Glo1 interacts with HUWE1 but not with other E3 ubiquitin ligases increased in $\mathrm{HG}$ concentration cultures in this study-CHIP and ring finger protein- $31 .{ }^{49}$ Increased HUWE1 may, therefore, mediate increased ubiquitination, proteolysis and downregulation of Glo1 protein in
HG concentration cultures and thereby exacerbate dicarbonyl stress. This remains to be experimentally validated in future studies.

There is increasing evidence that Glo1 inducer, tRESHESP, is an effective treatment to correct dicarbonyl stress in vitro and clinically. ${ }^{12}{ }^{19}$ Herein, tRES-HESP increased the activity of Glo1 in LG concentration cultures and corrected the decrease of Glo1 activity in HG concentration cultures. tRES-HESP increases Glo1 expression by activation and binding of $\mathrm{Nrf2}$ to a regulatory ARE in the GLO1 gene. ${ }^{18} 19$ It also decreased the flux of formation of D-lactate and consumption of glucose in LG concentration cultures and corrected the increased flux of formation of D-lactate and glucose consumption in HG concentration cultures. With tRES-HESP treatment, therefore, PDLFs exposed to HG concentration maintain normal glucose metabolism. This is expected to correct all dysfunction and disturbance of the cytosolic proteome. We have previously identified how this occurs: tRES-HESP 
increases expression of glucose-6-phosphate dehydrogenase (G6PD) through ARE-linked gene expression; this decreases the cellular concentration of glucose-6-phosphate (G6P) and impairs functionality of Mondo A/Mlx/G6P complex, decreasing carbohydrate response element-linked expression of HK2. ${ }^{12}$ No effects of tRES-HESP was found on activity of Glo2 and MG reductase and MG dehydrogenase.

Interestingly, in previous studies, tRES-HESP also corrected metabolic dysfunction of bone marrow progenitor cell in experimental diabetes, improving wound closure and angiogenesis in diabetic mice. This involved correction of the UPR stress sensor of the ER, inositol requiring enzyme $1 \alpha .{ }^{50}$

tRES-HESP was optimized for induction of expression of Glo1 by activation of Nrf2 with median effective concentration $\mathrm{EC}_{50}$ of $1.46 \mu \mathrm{M} .{ }^{19}$ Other Nrf2-regulated genes are implicated in the prevention of metabolic dysfunction induced by HG concentration in PDLFs in culture: increased expression of G6PD (see above) and $\gamma$-glutamylcysteine ligase, catalytic and modifier subunits $(\gamma$-glutamylcysteine ligase and $\gamma$-glutamylcysteineligase, modifier subunit)-likely mediating the increase in cellular GSH in both LG and HG concentration cultures. ${ }^{19}$ In the activation of Nrf2 by tRES and HESP in combination, we implicated upstream activation of AMP kinase and sirtuin-1-through inhibition of cAMP phosphodiesterase by tRES and activation of protein kinase A by HESP $^{51}$ — which are also expected to improve dysglycemia in vivo, as reviewed. ${ }^{52} 53$ Nevertheless, by decreasing expression of HK2 and correcting increased glucose metabolism in PDLFs in HG concentration cultures, tRES-HESP cuts off the driver for metabolic function at source. ${ }^{1112}$ Other effects may be dependent or of secondary importance to this.

To assess functional effects of Glo1 inducers on PDLFs, we measured binding of PDLFs to collagen-I. Increased physiological levels of MG may drive PDLF dysfunction and detachment from the extracellular matrix-as found for vascular endothelial cells in HG cultures. ${ }^{16}$ This is particularly damaging for sustaining the PDL. Surprisingly, tRES-HESP increased the binding of PDLFs to collagen in cultures with both LG and HG concentration, correcting the decreased collagen-I binding of PDLFs in HG concentrations cultures. Previous studies have shown that MG targets functional RGD and GFOGER integrin binding sites in the extracellular matrix and stimulates cell detachment ${ }^{16}$ - the latter mediating binding of PDLFs to collagen-I. ${ }^{8}$ Also, overexpression of Glo1 in endothelial cells in HG concentration cultures improved extracellular matrix binding, ${ }^{54}$ suggesting that there are likely both benefits of decreased extracellular matrix target modification and improved cell function by correcting dicarbonyl stress-such as maintaining integrin function mediating this response. Impaired PDLF-extracellular matrix interaction in dicarbonyl stress may explain tissue breakdown of periodontium in periodontal diseases in hyperglycemia. Administration of tRES to a rat model of periodontitis produced inhibition of periodontitismediated loss of alveolar bone and tissue breakdown in the periodontium.$^{55}$ tRES-HESP combination has benefits over tRES alone in vivo through synergism at the pharmacological target, Nrf2, and also likely improved bioavailability of tRES through HESP-mediated inhibition of intestinal glucuronosyl-transferases. ${ }^{51}$ It is a candidate for investigational treatment for periodontitis in diabetes.

\section{Author affiliations}

${ }^{1}$ Speciality Clinics, University Dental Hospital, Taif Dental College, Taif University, Taif, Saudi Arabia

${ }^{2}$ Clinical Sciences Research Laboratories, Warwick Medical School, University of Warwick, University Hospital, Coventry, UK

${ }^{3}$ Diabetes Research Center, Qatar Biomedical Research Institute, Hamad Bin Khalifa University, Qatar Foundation, Doha, Qatar

${ }^{4}$ College of Health and Life Sciences, Hamad Bin Khalifa University, Qatar Foundation, Doha, Qatar

${ }^{5}$ Department of Basic Medical Science, College of Medicine, QU Health, Qatar University, Doha, Qatar

Acknowledgements The authors would like to thank Dr M. Tauqeer Alam (Warwick Medical School and Zeeman Institute, University of Warwick) for discussions on protein bioinformatics and Dr Alaa Shafie (Department of Medical Laboratories, Taif University, KSA) for discussions on PDLF culture studies.

Contributors AA cultured and analyzed cells for glyoxalase system, glucose metabolism and proteomics analysis; MX performed some sample analysis; NR and PJT analyzed the proteomics data; MA-M and PJT performed the bioinformatics analysis and PJT and NR obtained the funding, designed the study, analyzed the data and wrote the manuscript. The final manuscript has been reviewed and approved by all authors.

Funding This study was funded by the Ministry of Education, Saudi Arabia, for a PhD studentship to AA, Qatar Foundation for a PhD studentship to MA-M and funding for PJT's research and Qatar University for funding research to NR.

Competing interests None declared.

Patient consent for publication Not required.

Ethics approval All methods were carried out in accordance with relevant guidelines and regulations and all experimental protocols were approved by University of Warwick Genetic Modification \& Biosafety Committee (Project no. 305). Local ethics approval was not required as the human PDLFs were provided, after prior passage in vitro, by a commercial supplier.

Provenance and peer review Not commissioned; externally peer reviewed.

Data availability statement All data are available on reasonable request directed to the Lead Contact, NR (n.rabbani@qu.edu.qa).

Supplemental material This content has been supplied by the author(s). It has not been vetted by BMJ Publishing Group Limited (BMJ) and may not have been peer-reviewed. Any opinions or recommendations discussed are solely those of the author(s) and are not endorsed by BMJ. BMJ disclaims all liability and responsibility arising from any reliance placed on the content. Where the content includes any translated material, BMJ does not warrant the accuracy and reliability of the translations (including but not limited to local regulations, clinical guidelines, terminology, drug names and drug dosages), and is not responsible for any error and/or omissions arising from translation and adaptation or otherwise.

Open access This is an open access article distributed in accordance with the Creative Commons Attribution Non Commercial (CC BY-NC 4.0) license, which permits others to distribute, remix, adapt, build upon this work non-commercially, and license their derivative works on different terms, provided the original work is properly cited, appropriate credit is given, any changes made indicated, and the use is non-commercial. See: http://creativecommons.org/licenses/by-nc/4.0/.

\section{ORCID iDs}

Paul J Thornalley http://orcid.org/0000-0001-7659-443X

Naila Rabbani http://orcid.org/0000-0002-5819-2506 


\section{REFERENCES}

1 Pihlstrom BL, Michalowicz BS, Johnson NW. Periodontal diseases. The Lancet 2005;366:1809-20.

2 American Diabetes Association. 3. Comprehensive Medical Evaluation and Assessment of Comorbidities: Standards of Medical Care in Diabetes-2018. Diabetes Care 2018;41:S28-37.

3 Michalowicz BS, Diehl SR, Gunsolley JC, et al. Evidence of a substantial genetic basis for risk of adult periodontitis. $J$ Periodontol 2000;71:1699-707.

4 Van Dyke TE, Serhan CN. Resolution of inflammation: a new paradigm for the pathogenesis of periodontal diseases. J Dent Res 2003;82:82-90.

5 Brownlee M. Biochemistry and molecular cell biology of diabetic complications. Nature 2001;414:813-20.

6 de Jong T, Bakker AD, Everts V, et al. The intricate anatomy of the periodontal ligament and its development: lessons for periodontal regeneration. J Periodontal Res 2017;52:965-74.

7 Sodek J, McKee MD. Molecular and cellular biology of alveolar bone. Periodontol 2000 2000;24:99-126.

8 Barczyk M, Bolstad Al, Gullberg D. Role of integrins in the periodontal ligament: organizers and facilitators. Periodontol 2000 2013;63:29-47.

9 Nishimura F, Takahashi K, Kurihara M, et al. Periodontal disease as a complication of diabetes mellitus. Ann Periodontol 1998;3:20-9.

10 El-Zainy MA, Halawa AM, Saad FA. Effect of diabetes mellitus on cementum periodontal interface in streptozotocin-induced diabetic rat model. Future Dental Journal 2018;4:181-8.

11 Rabbani N, Thornalley PJ. Hexokinase-2 glycolytic overload in diabetes and ischemia-reperfusion injury. Trends Endocrinol Metab 2019;30:419-31.

12 Irshad Z, Xue M, Ashour A, et al. Activation of the unfolded protein response in high glucose treated endothelial cells is mediated by methylglyoxal. Sci Rep 2019;9:7889.

13 Rabbani N, Xue M, Thornalley PJ. Methylglyoxal-Induced dicarbonyl stress in aging and disease: first steps towards glyoxalase 1-based treatments. Clin Sci 2016;130:1677-96.

14 Phillips SA, Thornalley PJ. The formation of methylglyoxal from triose phosphates. investigation using a specific assay for methylglyoxal. Eur J Biochem 1993;212:101-5.

15 Ahmed N, Dobler D, Dean M, et al. Peptide mapping identifies hotspot site of modification in human serum albumin by methylglyoxal involved in ligand binding and esterase activity. J Biol Chem 2005;280:5724-32.

16 Dobler D, Ahmed N, Song L, et al. Increased dicarbonyl metabolism in endothelial cells in hyperglycemia induces anoikis and impairs angiogenesis by RGD and GFOGER motif modification. Diabetes 2006;55:1961-9.

17 Murillo J, Wang Y, Xu X, et al. Advanced glycation of type I collagen and fibronectin modifies periodontal cell behavior. $J$ Periodontol 2008;79:2190-9.

18 Xue M, Rabbani N, Momiji H, et al. Transcriptional control of glyoxalase 1 by Nrf2 provides a stress-responsive defence against dicarbonyl glycation. Biochem J 2012;443:213-22.

19 Xue M, Weickert MO, Qureshi S, et al. Improved glycemic control and vascular function in overweight and obese subjects by glyoxalase 1 inducer formulation. Diabetes 2016;65:2282-94.

20 Mariotti A, Cochran DL. Characterization of fibroblasts derived from human periodontal ligament and gingiva. $J$ Periodontol 1990;61:103-11.

21 Somerman MJ, Young MF, Foster RA, et al. Characteristics of human periodontal ligament cells in vitro. Arch Oral Biol 1990;35:241-7.

22 McLellan AC, Thornalley PJ. Synthesis and chromatography of 1,2-diamino-4,5-dimethoxybenzene, 6,7-dimethoxy-2methylquinoxaline and 6,7-dimethoxy-2,3-dimethylquinoxaline for use in a liquid chromatographic fluorimetric assay of methylglyoxal. Anal Chim Acta 1992;263:137-42.

23 Shafie A, Xue M, Barker G, et al. Reappraisal of putative glyoxalase 1-deficient mouse and dicarbonyl stress on embryonic stem cells in vitro. Biochem J 2016;473:4255-70.

24 Rabbani N, Thornalley PJ. Measurement of methylglyoxal by stable isotopic dilution analysis LC-MS/MS with corroborative prediction in physiological samples. Nat Protoc 2014;9:1969-79.

25 Rabbani N, Shaheen F, Anwar A, et al. Assay of methylglyoxalderived protein and nucleotide ages. Biochem Soc Trans 2014;42:511-7.

26 Ellman GL. Tissue sulfhydryl groups. Arch Biochem Biophys 1959;82:70-7.

27 Rabbani N, Ashour A, Thornalley PJ. Mass spectrometric determination of early and advanced glycation in biology. Glycoconj J 2016;33:553-68.
28 Huang DW, Sherman BT, Lempicki RA. Systematic and integrative analysis of large gene Lists using David bioinformatics resources. Nat Protoc 2009;4:44-57.

29 Gallet X, Charloteaux B, Thomas A, et al. A fast method to predict protein interaction sites from sequences. $J \mathrm{Mol} B i \mathrm{O}$ 2000;302:917-26.

30 Abikshyeet P, Ramesh V, Oza N. Glucose estimation in the salivary secretion of diabetes mellitus patients. Diabetes Metab Syndr Obes 2012;5:149-54.

31 Mitsui Y, Schneider EL. Relationship between cell replication and volume in senescent human diploid fibroblasts. Mech Ageing Dev 1976;5:45-56.

32 McLellan AC, Thornalley PJ, Benn J, et al. Glyoxalase system in clinical diabetes mellitus and correlation with diabetic complications. Clin Sci 1994:87:21-9.

33 Rabbani N, Xue M, Thornalley PJ. Activity, regulation, copy number and function in the glyoxalase system. Biochem Soc Trans 2014:42:419-24.

34 Thornalley PJ, Yurek-George A, Argirov OK. Kinetics and mechanism of the reaction of aminoguanidine with the alpha-oxoaldehydes glyoxal, methylglyoxal, and 3-deoxyglucosone under physiological conditions. Biochem Pharmacol 2000;60:55-65.

35 Longo N, Bell Gl, Shuster RC, et al. Human fibroblasts express the insulin-responsive glucose transporter (GLUT4). Trans Assoc Am Physicians 1990;103:202-13.

36 Hexokinase TT. Allosteric regulatory enzymes. Boston, MA: Springer US, 2008: 179-98

37 Kohda Y, Iwatate K, Tanaka T, et al. Metabolic fate of excessive glucose in fibroblast cells in a diabetic setting. Fundam Toxicol Sci 2015;2:55-60.

38 Pang L, Wang Y, Zheng M, et al. Transcriptomic study of high-glucose effects on human skin fibroblast cells. Mol Med Rep 2016;13:2627-34.

39 Xia H-G, Najafov A, Geng J, et al. Degradation of hK2 by chaperonemediated autophagy promotes metabolic catastrophe and cell death. J Cell Biol 2015;210:705-16.

40 Chang Y-C, Hsieh Y-S, Lii C-K, et al. Induction of C-fos expression by nicotine in human periodontal ligament fibroblasts is related to cellular thiol levels. J Periodontal Res 2003;38:44-50.

41 Shelton MD, Chock PB, Mieyal JJ. Glutaredoxin: role in reversible protein S-glutathionylation and regulation of redox signal transduction and protein translocation. Antioxid Redox Signal 2005;7:348-66.

42 Shelton MD, Kern TS, Mieyal JJ. Glutaredoxin regulates nuclear factor kappa-B and intercellular adhesion molecule in Müller cells: model of diabetic retinopathy. J Biol Chem 2007;282:12467-74.

43 Aesif SW, Kuipers I, van der Velden J, et al. Activation of the glutaredoxin- 1 gene by nuclear factor $\mathrm{KB}$ enhances signaling. Free Radic Biol Med 2011;51:1249-57.

44 Neef DW, Jaeger AM, Gomez-Pastor R, et al. A direct regulatory interaction between chaperonin TRiC and stress-responsive transcription factor HSF1. Cell Rep 2014:9:955-66.

45 Lee J, Ozcan U. Unfolded protein response signaling and metabolic diseases. J Biol Chem 2014;289:1203-11.

46 Cole NB, Ellenberg J, Song J, et al. Retrograde transport of Golgilocalized proteins to the ER. J Cell Biol 1998;140:1-15.

47 Kriegenburg F, Ellgaard L, Hartmann-Petersen R. Molecular chaperones in targeting misfolded proteins for ubiquitin-dependent degradation. Febs J 2012;279:532-42.

$48 \mathrm{Xu}$ Y, Anderson DE, Ye Y. The HECT domain ubiquitin ligase HUWE1 targets unassembled soluble proteins for degradation. Cell Discov 2016;2:16040-40.

49 Rouillard AD, Gundersen GW, Fernandez NF, et al. The harmonizome: a collection of processed datasets gathered to serve and mine knowledge about genes and proteins. Database 2016;2016:baw100.

$50 \mathrm{Li} \mathrm{H}$, O'Meara M, Zhang X, et al. Ameliorating methylglyoxal-induced progenitor cell dysfunction for tissue repair in diabetes. Diabetes 2019;68:1287-302.

51 Rabbani N, Thornalley PJ. Glyoxalase 1 modulation in obesity and diabetes. Antioxid Redox Signal 2019;30:354-74.

52 Steinberg GR. Cellular energy sensing and Metabolism-Implications for treating diabetes: the 2017 outstanding scientific achievement Award Lecture. Diabetes 2018;67:169-79.

53 Nogueiras R, Habegger KM, Chaudhary N, et al. Sirtuin 1 and sirtuin 3: physiological modulators of metabolism. Physiol Rev 2012;92:1479-514.

54 Ahmed U, Dobler D, Larkin SJ, et al. Reversal of hyperglycemiainduced angiogenesis deficit of human endothelial cells by overexpression of glyoxalase 1 in vitro. Ann NY Acad Sci 2008;1126:262-4.

55 Bhattarai G, Poudel SB, Kook S-H, et al. Resveratrol prevents alveolar bone loss in an experimental rat model of periodontitis. Acta Biomater 2016;29:398-408. 